\title{
EASY-PLANE MAGNETIC ANISOTROPY INDUCED BY STRESS-ANNEALING IN FINEMET-TYPE MATERIALS
}

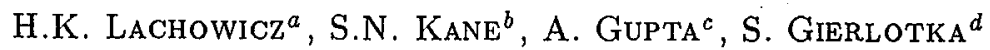 \\ AND B. PAEOSZ ${ }^{d}$ \\ ${ }^{a}$ Institute of Physics, Polish Academy of Sciences \\ Al. Lotników 32/46, 02-668 Warszawa, Poland \\ ${ }^{b}$ School of Physics, Devi Ahilya University \\ Khandwa Road, Indore (M.P.), 452001 India \\ 'Inter-University Consortium for DAEF \\ University Campus, Khandwa Road, Indore, India \\ ${ }^{d}$ High Pressure Research Center, Polish Academy of Sciences \\ Sokołowska 29, 00-901 Warszawa, Poland
}

(Received February 13, 1998)

It is shown that annealing under stress of FINEMET-type metallic glass ribbon (FeCuNbSiB) induces magnetic anisotropy of an easy-plane type (cross-section of the ribbon). This conclusion has been drawn analyzing experimental results obtained by means of Kerr-effect (domain structure) and Mössbauer spectroscopy operating in the so-called "magic angle" configuration which allows us to calculate all three spatial components of magnetization. Additionally, it is also shown that no crystallographic texture is created in the sample after stress-annealing, the feature which would have been an origin of the observed anisotropy.

PACS numbers: $75.30 . \mathrm{Gw}, 75.50 . \mathrm{Kj}, 75.60 . \mathrm{Ch}$

\section{Introduction}

It is well known that application of stress during crystallization of FINEMET-type ( $\mathrm{FeCuNbSiB}$ ) amorphous parent alloys produced in a form of ribbon by rapid quenching, creates giant anisotropy of the magnitude of the order of $10^{3} \mathrm{~J} / \mathrm{m}^{3}$ (see, e.g. [1]). This anisotropy is mainly generated within the volume of the nanocrystalline phase as it has explicitly been proved experimentally (see, e.g. [2]). Two mechanisms were taken into consideration as the origin of the stress-anneal-induced anisotropy. Herzer [3] proposed an explanation claiming that this anisotropy is of magnetoelastic nature and is created in the nanocrystallites (bcc-FeSi grains) due to tensile back stresses exerted by the anelastically deformed residual amorphous matrix. Herzer has drawn this conclusion because of a strong correlation between the stress-induced anisotropy and the magnetostriction of the nanocrystallites found by him. Afterwards, Hofmann and Kronmüller [4] suggested an alter- 
native explanation of the origin of the considered anisotropy. They have adapted Neel's calculations of diatomic directional ordering to the conditions of the studied conventional FINEMET-alloy, obtaining quite satisfactory agreement with the experimental data. Recently, Lachowicz et al. [2] confirmed the above conclusion showing that the anisotropic ordering of atomic pairs seems to be the most probable origin of the studied anisotropy in FINEMET-type nanocrystalline materials. They have arrived at this conclusion analyzing temperature dependences of the stress-anneal-induced anisotropy.

The stress-annealing of FINEMET-type materials usually creates a magnetically hard axis along the direction of stress, i.e. along the ribbon length. In the direction transverse to this axis but lying in the plane of the sample, magnetization of the sample proceeds in a much easier way. Because of this, the stress-anneal-induced anisotropy is usually perceived as the uniaxial anisotropy with its easy axis of magnetization directed transverse to the ribbon length. In fact, this anisotropy is of the easy-plane type (cross-section of the ribbon), similarly as the stress-anneal-induced anisotropy generated in metallic glasses as it has clearly been interpreted by Závěta et al. [5] years ago.

The aim of the present work is to prove experimentally that the anisotropy induced by stress in nanocrystalline FINEMET-type materials is indeed of the easy-plane type.

\section{Experimental}

For the experiments, FINEMET-type rapidly quenched amorphous ribbon of the composition $\mathrm{Fe}_{73.5} \mathrm{Cu}_{1} \mathrm{Nb}_{3} \mathrm{Si}_{15.5} \mathrm{~B}_{7}$ (produced by Vacuumschmelze, GmbH) was used as the parent material for the nanocrystalline samples. The ribbon was $6 \mathrm{~mm}$ wide and $23 \mu \mathrm{m}$ thick. Crystallization under tensile stress was carried on in a conventional tube furnace in its vertical position in an inert atmosphere of flowing helium to avoid surface oxidation of the samples. A weight was suspended at the cold bottom end of the ribbon to introduce stresses. The samples were first pre-annealed at $300^{\circ} \mathrm{C}$ for $1 \mathrm{~h}$ and next stress-annealed at $540^{\circ} \mathrm{C}$ again for $1 \mathrm{~h}$ (optimum annealing conditions for FINEMETs) with the stress of $213 \mathrm{MiPa}$. At these annealing conditions, the effective anisotropy was equal to $3100 \mathrm{~J} / \mathrm{m}^{3}$ as calculated from the room temperature hard-axis magnetization characteristics.

The microstructure of the annealed sample was inspected by TEM-microscopy and the obtained micrograph was used to estimate the average size of the crystallites which equals $\approx 20 \mathrm{~nm}$. Their volumetric content has also been estimated using magnetic method (for details see, e.g. [6]), showing that $\approx 70 \%$ of the volume of the samples is occupied by the nanocrystallites.

In order to prove that the stress-anneal-induced anisotropy is of the easy-plane type, magnetic domain structure in the sample has been observed. These observations have been carried on by means of Kerr-effect technique using a computerized setup which makes it possible to cancel nonmagnetic background. Before observation the sample was demagnetized by an a.c. field.

Mössbauer spectroscopy has also been used to analyze spatial distribution of the magnetization in the as-querrched sample as well as in the samples arnealed without stress and under stress. The so-called "magic angle" configuration was ap- 
plied to calculate this distribution from the spectra obtained at room temperature (for the details of this method, see, e.g. [7]).

One of the possible origin of the considered anisotropy may also be a texture of the nanocrystallites created at the time of annealing under stress which might force the grains to grow with preferential crystallographic orientation. Though, this effect has been excluded [8], nevertheless, no experimental data were presented in the literature to confirm this conclusion. To check it, X-ray diffraction technique using monochromatic $\mathrm{Cu} K_{\alpha}$ radiation (Siemens, D5000) has been used in this work to compare the spectra obtained for the samples annealed at the unloaded conditions and under stress.

\section{Results and discussion}

Figure 1 shows the highest intensity peaks $[(110)-\alpha$ - $\mathrm{Fe}(\mathrm{Si})]$ which appear in the X-ray diffraction patterns obtained for the sample annealed under unloaded conditions and for the sample annealed with the applied tensile stress of $213 \mathrm{MPa}$. Both samples were first preannealed at $300^{\circ} \mathrm{C}$ for $1 \mathrm{~h}$ and next isothermally heated at $540^{\circ} \mathrm{C}$ for $1 \mathrm{~h}$. The measurements of diffraction spectra were carried on for each sample in two configurations - incident X-ray along the sample axis (parallel configuration) and transverse to this axis (transverse geometry). Figure 1a presents two aforementioned peaks obtained for both samples at parallel configuration, whereas Fig. $1 \mathrm{~b}$ shows these peaks measured at transverse geometry. The observed superimposition of these peaks evidences that the distribution of crystallographic orientations is the very same in both samples and does not depend on the annealing conditions. This result explicitly shows that no texture is created by stress-annealing since the occurrence of texture in the sample annealed under stress should manifest itself in a change of intensity of the observed peak compared to that characteristics for the sample annealed under unloaded conditions. The additional conclusion which can be drawn from the above results is that the average size of the crystallites is the same in the sample annealed with and without stresses (the same width of the peaks).

Figure $2 a$ presents magnetic domain pattern observed on the surface of the stress-annealed sample (Fig. 2b shows an enlarged part of this pattern to make the shape of the domain walls better visible, fivefold magnification). As is seen in these figures, the structure consists of relatively wide stripe domains directed transversely to the ribbon axis but the walls separating them do not intersect the ribbon surface in a straight line but in a zig-zag shape meander.

The zig-zag shaped walls have been observed in the past in several crystalline conventional magnetic materials under various circumstances (see, e.g. [9]). Almost a decade ago Závěta et al. [5] noticed similarly shaped walls in stress-annealed metallic glasses, showing that such a zig-zag shape of the wall is attributed to the anisotropy of easy-plane type (cross-section of the ribbon). An observed subdivision of the Bloch wall at the surface of the sample into parts with alternately opposite chirality of the local magnetic moments, leads to the decrease in the magnetostatic energy, the phenomenon well-known in the past when studying the domain structure in thin films. The similarity of the domain structure in the examined sample with those observed in stress-annealed metallic glasses does show that at the 

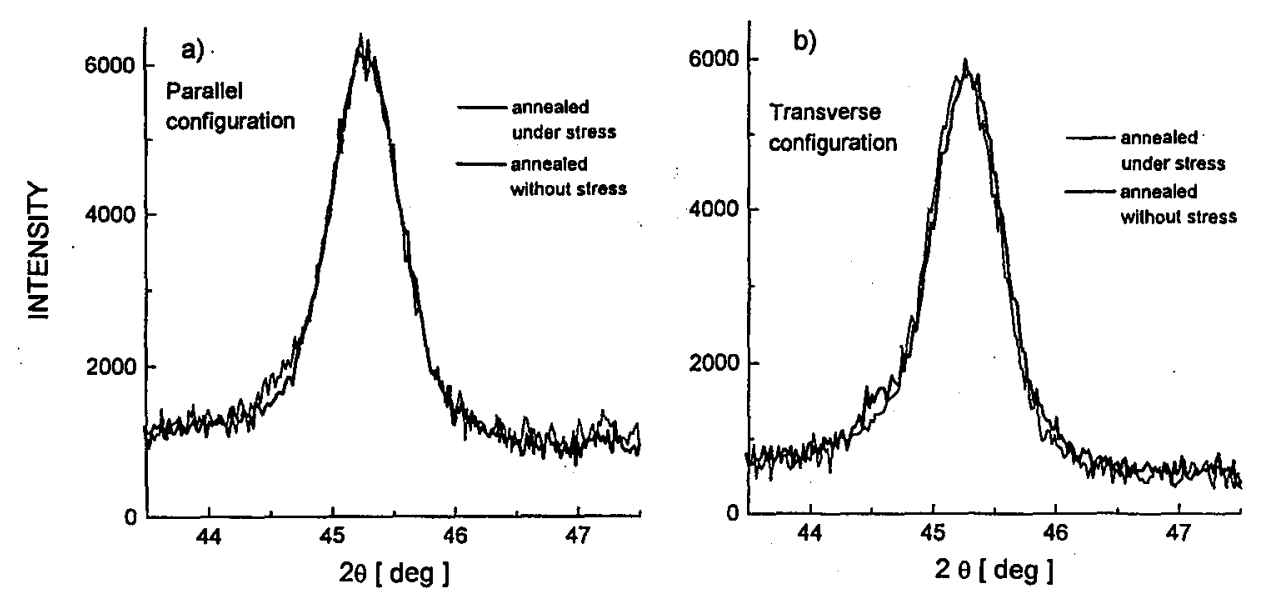

Fig. 1. The highest intensity peaks $[(110)-\alpha-\mathrm{Fe}(\mathrm{Si})]$ for samples of $\mathrm{FeCuNbSiB}$ metallic glass, stress-annealed and anmealed without stress, obtained by means of X-ray diffraction, (a) for parallel configuration, (b) for transverse configuration.

applied conditions of annealing, an easy-plane anisotropy with its hard-axis along the stress direction (the ribbon length) is created in FINEMET-type nanocrystalline magnets. The overall easy-axis of this anisotropy is then determined by the intersection of the anisotropy and demagnetizing easy-planes, as it has been pointed out in Ref. [5]. Taking into account the geometry of the ribbon (thickness to width ratio is very large; for the ribbon used this ratio equals 0.0038 ), it seems to be not surprising that the considered anisotropy, in fact of easy-plane type, is usually considered as the uniaxial one.

It can be expected that the creation of easy-plane anisotropy should considerably change the spatial distribution of magnetization characteristics for the sample annealed without stress. For the sample annealed under stress, one should anticipate an increase in $z$ and $y$ components of magnetization (transverse and perpendicular directions, respectively) with a simultaneous decrease in $x$ component (along the ribbon axis).

To measure all these components $\left(N_{x}, N_{y}, N_{z}\right.$, while $\left.N_{x}+N_{y}+N_{z}=1\right)$, transmission ${ }^{57} \mathrm{Fe}$ Mössbauer spectroscopy is an excellent tool since the intensities of the intermediate lines of the magnetic sextet are related to the angle between the direction of radiation and that of the hyperfine field. The above components (discrete spin populations) can then be deduced from three different Mössbauer spectra recorded in convenient configurations (the aforementioned "magic angle" geometry).

For this experiment, transmission ${ }^{57} \mathrm{Fe}$ Mössbauer technique in constant acceleration mode was used. The measurements were performed at room temperature in three different geometries allowing us to calculate all three components of magnetization. Three samples were used for this experiment, namely: as-quenched, annealed without stress and annealed under stress. The results obtained from the Mössbauer experiments by an analysis of the measured spectra with the help of 

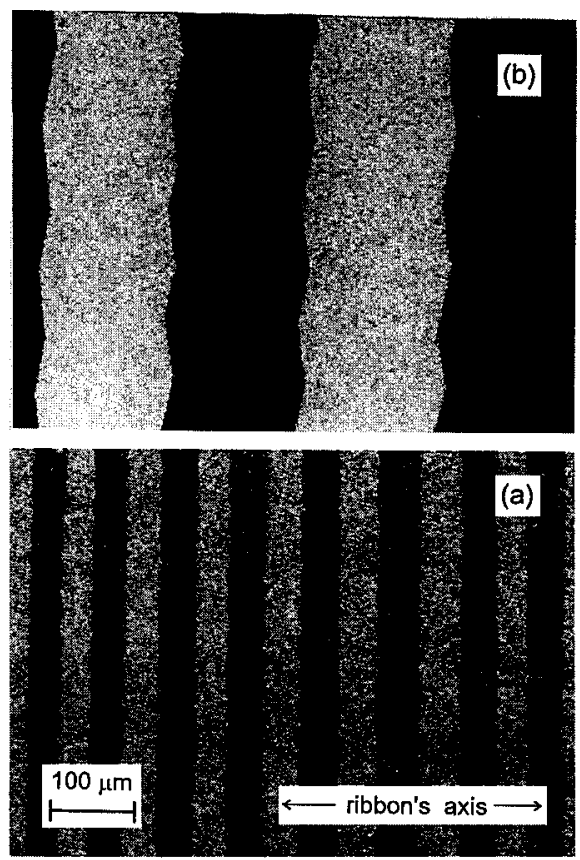

Fig. 2. Kerr-effect domain pattern observed on the surface of stress-annealed sample of $\mathrm{FeCuNbSiB}$ metallic glass sample (a), and an enlarged ( $\times 5)$ part of pattern shown in (a) to make zig-zags better visible.

NORMOS program [10], are presented in Table. Since all three components of magnetization were calculated individually using the experimental data obtained for three configurations of the measurements, in the last column in Table a sum of these components is given. It is seen that the error is in all cases not larger than $5 \%$, evidencing the reliability of the method. The components of magnetization behave according to the expectation as it is seen in this Table.

\section{TABLE}

Spatial components of magnetization (normalized) obtained by means of Mössbauer spectroscopy for as-quenched, annealed without stress and stress-annealed $\mathrm{FeCuNbSiB}$ metallic glass samples.

\begin{tabular}{l|c|c|c|c}
\hline \hline \multicolumn{1}{c|}{$N$ component } & $N_{x}$ & $N_{y}$ & $N_{z}$ & $N_{x}+N_{y}+N_{z}$ \\
\hline as-quenched & 0.296 & 0.385 & 0.291 & 0.972 \\
annealed at $540^{\circ} \mathrm{C} / 1 \mathrm{~h}$ & 0.424 & 0.413 & 0.209 & 1.001 \\
annealed at $540^{\circ} \mathrm{C} / 1 \mathrm{~h} / 213 \mathrm{MPa}$ & 0.046 & 0.633 & 0.322 & 1.046
\end{tabular}

For the amorphous sample (as-quenched-state) all three components of magnetization are of comparable values. The amorphous sample exhibits quite a large magnetostriction (around $20 \times 10^{-6}$ ) which interacting with the quenched-in stres- 
ses produces relatively large local magnetoelastic anisotropies of the accidental orientations of their easy-axes of magnetization. Considering the above, the nearly random distribution of magnetic moments is not surprising.

For the sample annealed without stress, the spatial distribution of the magnetization is mainly governed by the sample geometry (demagnetizing factors). In the created microstructural state of th' sample (fine crystallites embedded in the residual amorphous matrix), the magnetocrystalline anisotropy is averaged out and the sample exhibits relatively very low effective anisotropy (see, e.g. [11]). Additionally, magnetoelastic interactions become negligible because the quenched-in stresses are released by annealing and also because the sample in its nanocrystalline state is "zero"-magnetostrictive (magnetostriction lower than $10^{-6}[11]$ ).

After annealing under stress, a significant decrease in the $x$-component of magnetization is noticed with a simultaneous increase in both $y$ and $z$ components as should be expected if the easy-plane anisotropy is induced by stress-annealing.

\section{Conclusions}

An analysis of the results obtained using X-ray diffraction, observaticıs of domain structure and Mössbauer spectroscopy, show explicitly that annealing of FINEMET-type metallic glasses under tensile stress induces anisotropy of the easy-plane type (cross-section of the ribbon).

\section{Acknowledgments}

The work was partially supported by the Committee for Scientific Research (Poland) under research project No. 8 T11B 048 10. The authors are very grateful to Dr. G. Herzer (Vacuumschmelze, GmbH, Hanau, Germany) for supplying FINEMET-type metallic glass ribbon and to Mrs. T. Dragon (Max-Planck-Institute für Metallforschung, Stuttgart, Germany) for providing the domain structure photos.

\section{References}

[1] O.V. Nielsen, H.J.V. Nielsen, J. Magn. Magn. Mater. 22, 21 (1980).

[2] H.K. Lachowicz, A. Neuweiler, F. Popławski, E. Dynowska, J. Magn. Magn. Mater. 173, 287 (1997).

[3] G. Herzer, IEEE Trans. Magn. 30, 4800 (1994).

[4] B. Hofmann, H. Kronmüller, J. Magn. Magn. Mater. 152, 91 (1996).

[5] K. Závěta, L. Kraus, K. Jurek, V. Kamberský, J. Magn. Magn. Mater. 73, 334 (1988).

[6] A. Ślawska-Waniew ka, M. Gutowski, H.K. Lachowicz, T. Kulik, H. Matyja, Phys. Rev. B 46, 14594 (1992).

[7] G. LeGal, F. Varret, J. Magn. Magn. Mater. 111, 115 (1992).

[8] L. Kraus, K. Závěta, O. Heczko, P. Duhaj, G. Vlasák; J. Schneider, J. Magn. Magn. Mater. 112, 275 (1992).

[9] R. Gemperle, I. Tomáš, J. Magn. Magn. Mater. 73, 339 (1988).

[10] R.A. Brand, Nucl. Instrum. Methods Phys. Res. B 28, 398 (1987).

[11] G. Herzer, in: Handbook of Magnetic Materials, Ed. K.H.J. Buschow, Elsevier Science, Amsterdam 1997, p. 415. 